\title{
Mutual Information as a Measure of Contextual Effects on Single Switch Use
}

\author{
Negar Memarian ${ }^{1,2}$, Anastasios Venetsanopoulos $^{3,4}$ and Tom Chau ${ }^{*, 1,2}$ \\ ${ }^{I}$ Institute of Biomaterials and Biomedical Engineering, University of Toronto, Canada \\ ${ }^{2}$ Bloorview Research Institute, Bloorview Kids Rehab, Toronto, Ontario, Canada \\ ${ }^{3}$ Department of Electrical and Computer Engineering, University of Toronto, Canada \\ ${ }^{4}$ Department of Electrical and Computer Engineering, Ryerson University, Toronto, Canada
}

\begin{abstract}
Users with disability interact with augmentative and alternative communication devices, environmental control units, and computers via an access technology. While caregivers routinely exploit contextual information to interact meaningfully with individuals who are nonverbal and have severe motor impairments, access technologies to date have largely ignored context. Contextual factors include the environmental and personal factors in the model of functioning and disability introduced by The World Health Organization's International Classification of Functioning, Disability and Health in 2001.

We propose the use of mutual information as an objective means of measuring the impact of contextual factors on mechanical single switch usage. We show that common performance measures (e.g. sensitivity, specificity and response time) relating to switch use can be quantitatively unified within a mutual information measure. We exemplify the use of mutual information in the assessment of switch use in the presence of selected contextual factors. This information theoretic measure facilitates performance comparison amongst users and can potentially help in classification of contextual stimuli in terms of their impact (i.e. facilitating, barrier, neutral). Our examples with able-bodied participants and an individual with disability indicate that mutual information can be sensitive to changes in contextual factors. Mutual information may thus inform the design of individualized access technologies.
\end{abstract}

Keywords: Disability, rehabilitation, human-computer interaction, mutual information, contextual factors.

\section{INTRODUCTION}

\subsection{Access Pathways}

Individuals with severe and multiple disabilities often cannot employ conventional means of physical access, such as speech and gestures. An alternate channel is often required for communication and interaction with the environment. In rehabilitation terminology, that channel is termed an access pathway and constitutes the critical front end of an access solution [1]. From a system engineering perspective, an access solution is a system that receives a physical or physiological expression of the individual's intention as input. The system ultimately translates this input into a functional activity. A wide range of access pathways have been developed, from simple mechanical switches to sophisticated physiological ones. For a comprehensive review of emerging access technologies please see $[1,2]$.

\subsection{The Role of Context}

The usability of an access pathway is affected by both the personal characteristics of the user and the milieu in which the device is used [3]. In the World Health Organization's International Classification of Functioning, Disability and

*Address correspondence to this author at the Bloorview Research Institute, Bloorview Kids Rehab., 150 Kilgour Road, Toronto, M4G IR8, Canada; Tel: (416) 425-6220; Fax: (416) 425-1634;

E-mail: tom.chau@utoronto.ca
Health (ICF) [4], these personal and environmental characteristics are formally encapsulated into the concept of context, which is the collection of factors that define the physical, social, cultural and attitudinal environment within which people live their lives. Personal factors include age, sex, and indigenous status, personal resources (including physical and mental abilities), and personal perceptions [5] while environmental factors consist of the built environment, ambient temperature, time of day, air quality, and ambient noise, among other characteristics of the surrounding milieu.

The ICF recognizes that contextual factors and their interactions can influence the health domains of activity and participation. It is therefore not surprising that participation is measured in terms of performance in the individual's typical environment [6]. Likewise, it is believed that while the physical and cognitive ability to use an access pathway is important, primary emphasis should be placed on arranging favorable circumstances to enable the most effective and efficient device usage [7]. Indeed, a contextual factor may facilitate the process of working with an access pathway, hinder the process, or have no significant effect. For example, the presence of people may distract the user and hence become a hindering contextual factor. On the other hand, prior knowledge of the task at hand may help the user to anticipate future interactions and hence serve as a facilitating factor. Identifying the type and magnitude of impact of contextual factors may eventually lead to the development of 
context-aware access pathways. Such systems can potentially sense and compensate for the negative effects of certain hindering contextual factors or exploit facilitating factors to improve the robustness of an access pathway. However, access strategies developed to date do not account for personal and environmental factors and thus their usability declines when applied in more than one environment or by different users.

The importance of contextual factors in the delivery of assistive technology [8], functional assessments [9], measurement of participation [10-12], technology assessment [13], and modeling disability [14] has been recognized in the literature.

\subsection{Gauging Contextual Effects through Information Theory}

Based on the discussion in the previous section it therefore appears worthwhile to quantify the impact of contextual factors on the effectiveness of a given access pathway. In particular, an information theoretic approach may be useful, since access pathways, like other human-machine interfaces, involve the transmission of information. The combination of cognitive and physical abilities of the human user constitutes the communication channel through which the information is transmitted. The characterization of the human being as a communication channel is a nontrivial challenge [15]. For many years, quantitative models of information transmission in humans have been a subject of interest in fields such as psychology and human-computer interaction (HCI). In the 1950 s, soon after C.E. Shannon proposed information theory and the idea of mutual information in his famous 1948 paper [16], many psychologists tried to determine maximum information transmission rates in humans for various tasks, such as choice-reaction [17, 18], perception and learning [19], speed-accuracy of motor responses [20], vigilance [21] and recognition memory [22-24]. Studies show that the rate of information transmission in humans is affected by factors such as the dimensionality of the stimulus, the probability of stimulus occurrence and the context in which the stimulus occurs $[25,26]$.

Several groups have made use of information theory principles to model human-computer interaction. More than half a decade ago, Hick [17] and Hyman [18] published their findings from several choice-reaction experiments. The underlying theme of the ensuing Hick-Hyman law was that response time is not only a function of the number of stimulus alternatives but can also be considered a linear function of stimulus information (entropy). This finding spawned a number of attempts to design optimal control and display codes for human-computer interaction [27-29] although others have remarked that there has been limited uptake of these early concepts [30]. Chan \& Childress crafted theoretical relationships between human-machine noise and humanmachine output velocity [31], formulating the channel capacity of a human-machine system. They applied this formulation to estimate information transmission rates in human pursuit tracking [32]. On a separate front, Ogawa evaluated computer usability with a human-to-computer information transmission model [33] while Poock and Blackstone quantified the effectiveness of an augmentative and alternative communication (AAC) display by measuring input (the symbols requested by the clinician) and output (the responses given by the AAC user) entropies from which they estimated the relative information transmitted [34]. Recently, Sanger and Henderson optimized the graphical layout of an AAC device using a model of information rate and channel capacity that exploited the relationship between movement time and the number of buttons per screen, the size of the buttons, and the number of sequential button presses per word [35].

Building upon previous research, the objective of this paper is to demonstrate that contextual effects on a single switch access pathway can be meaningfully ascertained by estimating the rate of information transmission in the human communication channel. Using data from human participants, we illustrate a number of ways in which mutual information could be used to quantify switch usage within an experimental setting. This mutual information framework may inform the design of access pathways. Before we present our proposed framework however, we review the concept of mutual information in the case of transmitting binary information through a communication channel.

\subsection{Mutual Information in Transmission of Binary In- formation}

From an information theory perspective, the amount of information conveyed by a message from a source is measured by entropy. The more we know about the message from the source, the less the uncertainty or entropy, and the less the amount of information [36]. From information theory, we also know that in order to transmit information, there needs to be a transmitter (sender) and a receiver. The medium used to convey information from the transmitter to the receiver is called the communication channel.

We consider the formulation of mutual information in the case of transmitting binary information through a communication channel, that is, when there are only two message choices at any given time. An example of this communication scenario is a single switch, which is often used as the access pathway to derive a user interface. The switch can be either on (closed) or off (open). Let $X=\left\{x_{1}, x_{2}\right\}$ represent the messages that the transmitter sends, i.e., $x_{1}=\mathrm{ON}, x_{2}=\mathrm{OFF}$. Similarly, let $Y=\left\{y_{1}, y_{2}\right\}$ represent the messages that reach the receiver, i.e., $y_{1}=\mathrm{ON}, y_{2}=\mathrm{OFF}$. From information theory [37], the entropy of the transmitter (that is the rate at which the message source generates information) for the binary case is:

$$
H(X)=-\sum_{i=1}^{2} p\left(x_{i}\right) \log p\left(x_{i}\right) \text { bits per message }
$$

where $p\left(x_{i}\right)$ is the probability of transmitting message $i$.

Similarly at the receiver end the entropy is:

$$
H(Y)=-\sum_{j=1}^{2} p\left(y_{j}\right) \log p\left(y_{j}\right) \text { bits per message }
$$

where $p\left(y_{j}\right)$ is the probability of receiving message $j$.

In real communication channels, the transmitted and received messages may not coincide as a result of channel noise. The uncertainty as to which message was transmitted 
when a given message is received, is written as $H(X \mid Y)$ and is a natural measure of the information lost in transmission [37]. The quantity $H(X \mid Y)$ or the conditional entropy or equivocation of $X$ about $Y$ is estimated as

$$
\begin{aligned}
H(X \mid Y) & =\sum_{j=1}^{2} H\left(X \mid Y=y_{j}\right) p\left(y_{j}\right) \\
& =-\sum_{i=1}^{2} \sum_{j=1}^{2} p\left(x_{i} \mid y_{j}\right) p\left(y_{j}\right) \log p\left(x_{i} \mid y_{j}\right)
\end{aligned}
$$

bits per message

where $p\left(x_{i} \mid y_{j}\right)$ is the probability that message $i$ was transmitted given that message $j$ was received. Conditional entropy depends on how often $X$ is transmitted, or how often $Y$ is received, as well as on the errors made in transmission.

If we take $H(X)$ and $H(X \mid Y)$ as entropies in bits, then $I(X ; Y)$, the mutual information of $X$ and $Y$ is defined by [36]:

$$
I(X ; Y)=H(X)-H(X \mid Y)
$$

Equation (4a) can be written in terms of joint entropies as follows:

$$
\begin{aligned}
& I(X ; Y)=H(X)-[H(X, Y)-H(Y)] \\
& =H(X)+H(Y)-H(X, Y)
\end{aligned}
$$

The joint entropy $H(X, Y)$ is found from (5), in which $p\left(x_{i}, y_{j}\right)$ is the probability of occurrence of each pair of outcomes:

$$
H(X, Y)=-\sum_{i=1}^{2} \sum_{j=1}^{2} p\left(x_{i}, y_{j}\right) \log _{2}\left(p\left(x_{i}, y_{j}\right)\right)
$$

Mutual information is the amount of information that we learn about $X$, by virtue of knowing $Y$, or put another way, it is the amount of information about $X$ that is transmitted through the channel [38]. The reader unfamiliar with information theory may refer to $[36,37,39-41]$ for further reading.

\section{MATERIALS AND METHODS}

\subsection{Proposed Framework for Evaluating Contextual Ef- fects on Single Switch Use}

Here we introduce an information theoretic interpretation of the interface between a human user and a single switch access pathway, as depicted in Fig. (1). The computer which presents the visual and auditory cues can be considered as the transmitter, and the single switch (a means of acknowledging the cues) can be thought of as the receiver. The user constitutes the communication channel between the transmitter and the receiver. The proposed arrangement resonates closely with the human-machine communication models suggested by [18] and [42]. The user's accuracy and response time, and hence the characteristics of the communication channel, are affected by external factors such as infor- mation presentation modality, ambient noise or time of day as well as the cognitive and physical resources invoked.

Consider that the transmitter (T) in Fig. (1) presents an auditory or visual cue to the user. We distinguish between actionable (transmit " 1 ") and non-actionable (transmit "0") cues, the former being those which ought to trigger a switch activation by the user, or equivalently produce a 1 at the receiver (R). Likewise, non-actionable cues should not produce a response from the user and hence the corresponding receiver data should be 0 .

\subsection{Estimating Mutual information}

With the above framework and equation (4b), mutual information can be estimated from the entropies $H(X), H(Y)$ and $H(X, Y)$. Based on signal detection theory in psychology [26], Fig. (2) summarizes all possible sender-receiver cue combinations for the framework described in Fig. (1). Rows of the table correspond to cues presented to the user (transmitted cues, analogous to $x_{i}$ in equation (1)), while the columns represent cues acknowledged by the user (received cues, analogous to $y_{j}$ in equation (2)). True positives (TP) indicate the number of transmitted actionable cues that the user correctly acknowledged. False negatives (FN) indicate the number of transmitted actionable cues that were erroneously missed by the user. False positives (FP) indicate the number of non-actionable cues that the user erroneously acknowledged, and true negatives (TN) indicate the number of non-actionable cues that were correctly rejected by the user. The sum of all the cells is equal to the total number of messages displayed to the user, which we denote as $N$. The empirically estimated probabilities of the aforementioned events are [34]:

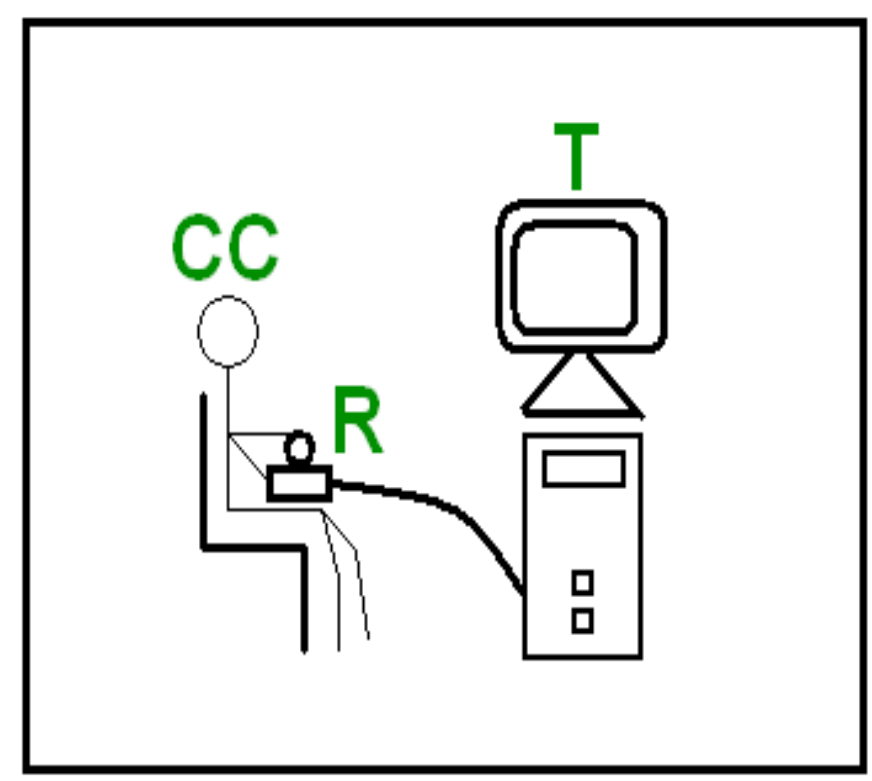

Fig. (1). Information-theoretic paradigm for single-switch access. A subject may react to visual, written or auditory stimuli by pressing a single mechanical switch. The computer, which generates the cues, can be considered the transmitter (T); the mechanical switch (a means of acknowledging the cues) can be thought of as the receiver $(\mathrm{R})$; the user constitutes the communication channel between the transmitter and the receiver (CC). 
$\mathrm{p}($ sending actionable cue $)=p\left(x_{1}\right)=\frac{T P+F N}{N}$

$\mathrm{p}($ sending non- actionable cue $)=p\left(x_{2}\right)=\frac{F P+T N}{N}$

$\mathrm{p}($ receiving actionable cue $)=p\left(y_{1}\right)=\frac{T P+F P}{N}$

$\mathrm{p}($ receiving non-actionable cue $)=p\left(y_{2}\right)=\frac{F N+T N}{N}$

p (sending actionable cue $\&$ receiving actionable cue)

$=p\left(x_{1}, y_{1}\right)=\frac{T P}{N}$

p (sending actionable cue $\&$ receiving non-actionable cue)

$=p\left(x_{1}, y_{2}\right)=\frac{F N}{N}$

p (sending non-actionable cue $\&$ receiving actionable cue)

$=p\left(x_{2}, y_{1}\right)=\frac{F P}{N}$

p (sending non-actionable cue \& receiving non-actionable cue $)=p\left(x_{2}, y_{2}\right)=\frac{T N}{N}$

In the present study, we obtained numerical estimates of mutual information by inserting the above probabilities into Equations (1) to (5). Mutual information represents the information shared between input $\mathrm{X}$ and output $\mathrm{Y}$, i.e. what the user is cued to do with the switch and what he/she actually does. When maximized over input distributions, mutual information gives us channel capacity.

\subsection{Participants}

Twelve able-bodied adults, aged $27.3 \pm 9.3$ years (six males) and a 29 year old male with $\mathrm{C} 1-\mathrm{C} 2$ incomplete spinal cord injury, participated in this study. Participants had no visual, auditory or cognitive impairments. Written consent was obtained from all participants.

\subsection{Protocol}

The protocol was approved by the Research Ethics Boards of Bloorview Kids Rehab and the University of To- ronto. The protocol consisted of a repeated trial of five different single-switch selection activities. Participants were presented with a series of visual or auditory cues (Table 1). In the first session, participants responded to the cues in a controlled environment. In the second session participants responded to the same cues but in the presence of a modified contextual factor. In each experiment outlined in Table 1, various visual or auditory messages were presented to the user, one at a time. Each message was presented for $600 \mathrm{~ms}$. Only a subset of messages were actionable cues, meaning that their presentation by the computer (transmitter) should ideally trigger a switch press by the user (receiver). For example in experiment 1(a), the actionable cue was a picture of a cat. Able-bodied participants were required to respond to actionable cues by pressing the spacebar on a computer keyboard. The participant with disability completed the tests by using a sip and puff switch. He was asked to respond to actionable cues with a puff, which was immediately translated to a left mouse click by means of a USB-based switch-toclick converter. The times of all switch activations as well as the time of presentation of actionable cues were automatically logged for subsequent off-line analysis.

It is important to note that the protocol was intended to exemplify different applications of the mutual information measure in gauging switch use context and not to conclusively determine specific contextual effects across populations.

\subsection{Data Collection and Analysis}

The logged stimulus presentation times, and switch activation times were used to calculate the number of TPs, FNs, FPs, and TNs. Each cue (regardless of being actionable or non-actionable) was presented to the user for 600 milliseconds. i.e. the cues changed every 0.6 second. In order to generate a TP, the user should have made switch activation within 600 milliseconds after onset of an actionable cue. No switch activation in this 600 millisecond interval translated to a miss or FN. A switch activation that occurred after the presentation of a non-actionable cue was recorded as a FP. A non-actionable cue correctly ignored by the user was considered a TN. With this data, mutual information was estimated according to section 2.2. In addition, sensitivity, specificity and average response time were calculated for each experiment. Average response time was calculated as the average
Cues acknowledged

by the user (received)

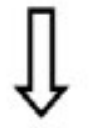

\section{Actionable Cue Non-actionable Cue}

\begin{tabular}{|c|c|c|c|}
\hline $\begin{array}{l}\text { Cues presented to the } \\
\text { user (transmitted) }\end{array}$ & $\begin{array}{l}\text { Actionable Cue } \\
\text { Non-actionable Cue }\end{array}$ & |True Positives (TP) & \\
\hline
\end{tabular}

Fig. (2). All possible sender-receiver cue combinations. 
Table 1. Summary of the Contextual Factors Explored in the Present Study and the Corresponding Experimental Procedures

\begin{tabular}{|c|c|c|}
\hline Experiment & Contextual Factor & Procedure \\
\hline 1 & Presentation modality & $\begin{array}{l}\text { The user is asked to respond by activating the switch upon observing a specific object (e.g., a cat) on the display } \\
\text { in three different trials: } \\
\text { (a) } 100 \text { pictures are presented to the user one at a time. The object of interest (i.e. picture of cat) appears at } \\
\text { some random points in the sequence } \\
\text { (b) } 100 \text { words are displayed to the user, one word at a time. The word of interest (i.e. cat) appears at some } \\
\text { random points in the sequence } \\
\text { (c) } 100 \text { words are spoken, one at a time, by the computer to the user. The word of interest (i.e. cat) is } \\
\text { announced at some random points in the sequence }\end{array}$ \\
\hline 2 & Prior knowledge & $\begin{array}{l}\text { The user is asked to perform the following two trials: } \\
\text { (a) A mixed series of } 100 \text { characters including letters (English alphabet all in caps), numbers and symbols are } \\
\text { displayed to the user, one at a time. The user is asked to activate the switch only when he/she observes a } \\
\text { letter of the alphabet. The user has no a priori knowledge of the next character in the sequence. } \\
\text { (b) Letters of the English alphabet (all in caps) are displayed to the user in order (Total of } 100 \text { letters). The user } \\
\text { is asked to activate the switch when he/she observes a vowel (i.e., 'A', 'E', 'I', 'O' and 'U'). }\end{array}$ \\
\hline 3 & Background noise & $\begin{array}{l}\text { A sequence of } 100 \text { pictures is displayed to the user. The user is asked to activate the switch when he/she observes } \\
\text { a specific object on the screen (e.g. a cat), while: } \\
\text { (a) The environment is quiet } \\
\text { (b) A source of noise is present in the environment (background conversations or music) }\end{array}$ \\
\hline 4 & Color & $\begin{array}{l}\text { The user is asked to perform the following two trials: } \\
\text { (a) } 100 \text { Uncolored shape outlines (circle, square, triangle, and rectangle) are displayed in random sequence to } \\
\text { the user, one at a time. The user is asked to activate the switch upon observing a specific shape (e.g. } \\
\text { square). } \\
\text { (b) } 100 \text { Shapes are displayed one at a time, in random sequence to the user. The user is asked to activate the } \\
\text { switch upon observing a specific shape with a specific color (e.g. purple square). All shapes are colorless } \\
\text { except the purple square and a purple cross that appear randomly in the sequence. }\end{array}$ \\
\hline 5 & $\begin{array}{l}\text { Presence of people in } \\
\text { the environment }\end{array}$ & $\begin{array}{l}\text { The user is asked to activate the switch upon hearing an auditory instruction while } \\
\text { (a) There is nobody except the examiner in the environment (controlled environment) } \\
\text { (b) There are people trespassing and chatting around the user (natural environment) }\end{array}$ \\
\hline
\end{tabular}

time it took the user to generate true positives.

\section{RESULTS}

The estimated mutual information for the five experiments are summarized by the box plots in Fig. (3). By visual inspection of these plots, we notice that mutual information may be influenced by certain contextual factors. A paired ttest revealed significant differences between various contextual conditions in Fig. (3a, b, e). To determine if these changes are statistically significant, one would need to systematically quantify the natural fluctuation in mutual information due to the inter-trial variability of human performance. We exemplify this statistical testing with one ablebodied participant. The participant repeated the experimental trials 15 times over the course of several days. Having confirmed that the 15 sets of mutual information were normally distributed (Kolmogorov- Smirnov test for normality), we used a paired samples t-test to compare mutual information between the baseline condition (no manipulation of contextual factors) and in the presence of a modified contextual factor. Results indicate that mutual information changed significantly between written and auditory presentation modalities $(t=-4.148, p=0.001)$. The corresponding mutual information probability density functions are shown in Fig. (4a). These densities were estimated from the mutual information of the 15 repeated trials, using a Gaussian mixture model.
One can visually verify the difference between written (dashed line) and auditory (dark solid line) presentation modalities. For the same individual, mutual information was also significantly lower over the 15 trials in the natural environment, likely due to the presence of other people $(t=2.386, p=0.032)$. This change in mutual information is depicted in Fig. (4b) by the shifted density function (dashed line) corresponding to the mutual information measured in the natural environment. Other contextual factors did not significantly change the mutual information of this particular participant.

Table 2 shows the mutual information for the participant with disability. Here, we exemplify the use of mutual information to gauge the effect of stimulus duration. $\mathrm{MI}_{600}$ and $\mathrm{MI}_{1000}$ denote the participant's mutual information when the stimulus presentation period was 600 and 1000 milliseconds, respectively. The participant with disability repeated the baseline condition (i.e., $600 \mathrm{~ms}$ stimulus duration) seven times to form baseline densities of mutual information. Using these baseline data and a one-sided, one-sample t-test, we statistically tested whether or not the $\mathrm{MI}_{1000}$ was statistically greater than the mean of the baseline distribution. In all cases, we found that indeed $\mathrm{MI}_{1000}>\mathrm{MI}_{600}(\mathrm{p}<0.05)$, implying that the longer stimulus duration is a facilitating factor for the individual with disability. 


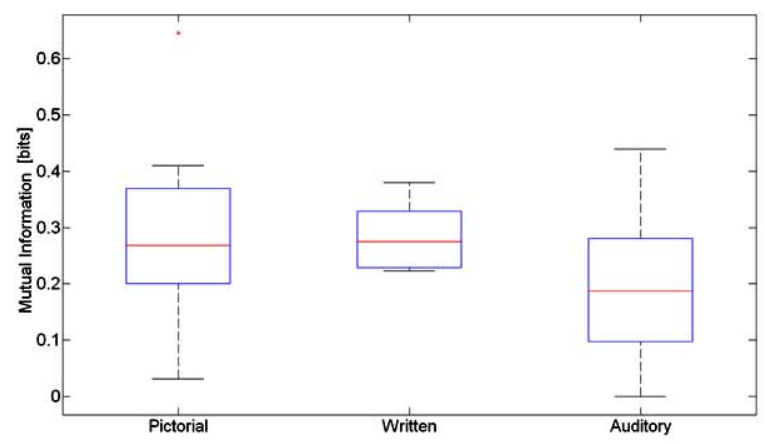

(a)

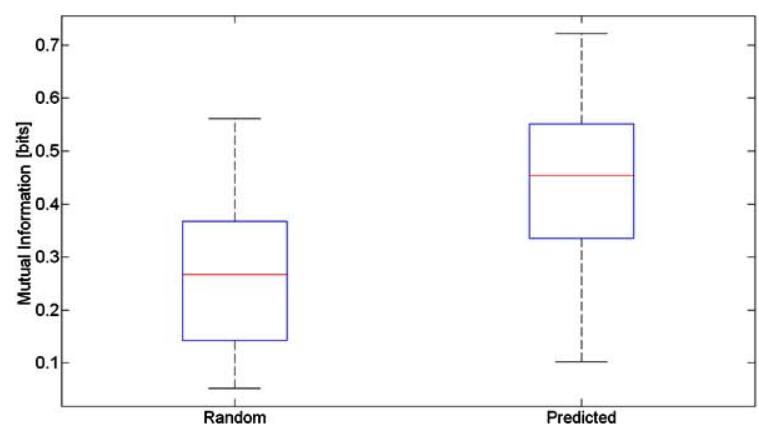

(b)

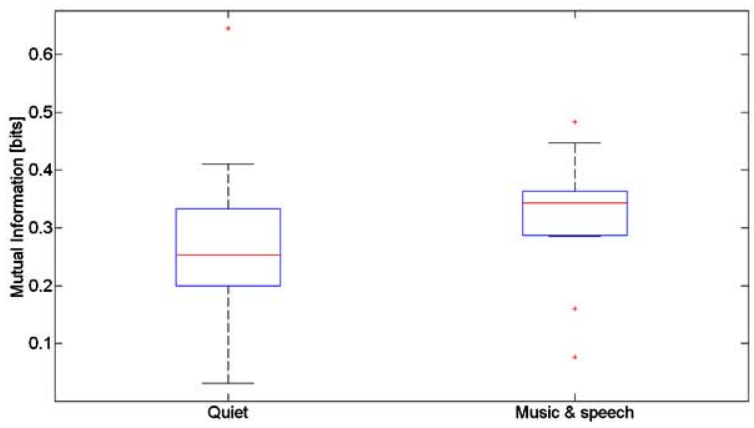

(c)

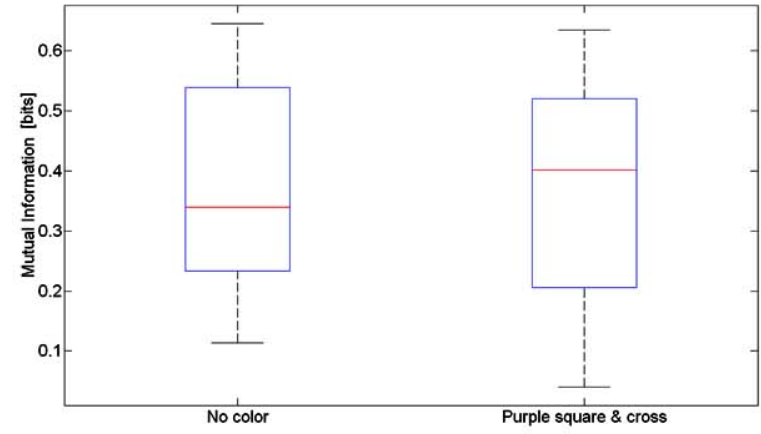

(d)

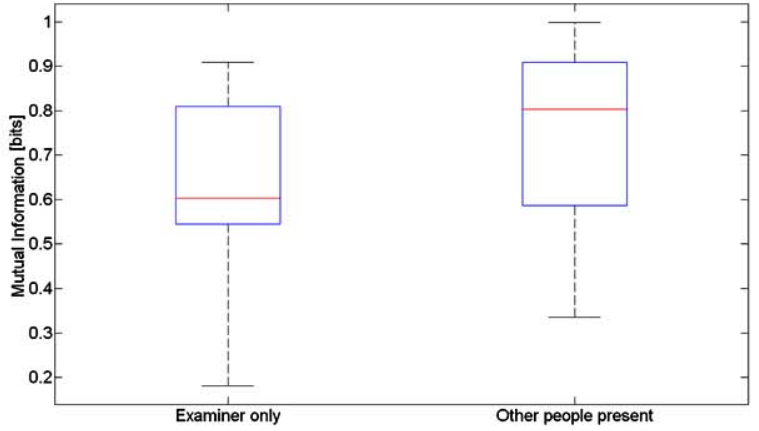

(e)

Fig. (3). Estimated mutual information of the twelve participants for the five experiments: (a) effect of presentation modality, (b) effect of prediction of choice, $(\mathbf{c})$ effect of ambient noise, (d) effect of color, (e) effect of presence of people in the environment. Graphs (a), (b) and (e) depict significant differences (paired t-test).

\section{DISCUSSION}

The mutual information measure provides a platform for quantitative assessment of contextual effects on single switch use. In particular it offers the following advantages over the conventional performance measures such as sensitivity and specificity.

\subsection{Contextual Factor Role Characterization}

Through the mutual information paradigm one can specify the type of effect of a particular contextual factor (i.e., facilitating, neutral, or hindering). From Fig. (5), we see that in response to various presentation modalities, $42 \%$ of the participants (participants 2, 3, 4, 6, and 11) exhibited the highest mutual information in response to written stimuli, while another $42 \%$ of the participants (participants 1, 7, 8, 10,12 ) had the highest mutual information in response to pictorial stimuli. Therefore we can infer that presenting the information in the written modality can be facilitating for the former group and information presentation in the pictorial modality can be facilitating for the latter group. Participants $3,6,7,8,11$, and 12 (50\% of participants) had their lowest mutual information in response to auditory stimuli, implying that the auditory modality is the least preferable for these participants. Message presentation exclusively by the auditory modality can thus be considered a hindering factor for these individuals. Participant 9 responded to pictorial and written modalities with comparable mutual information, im- 


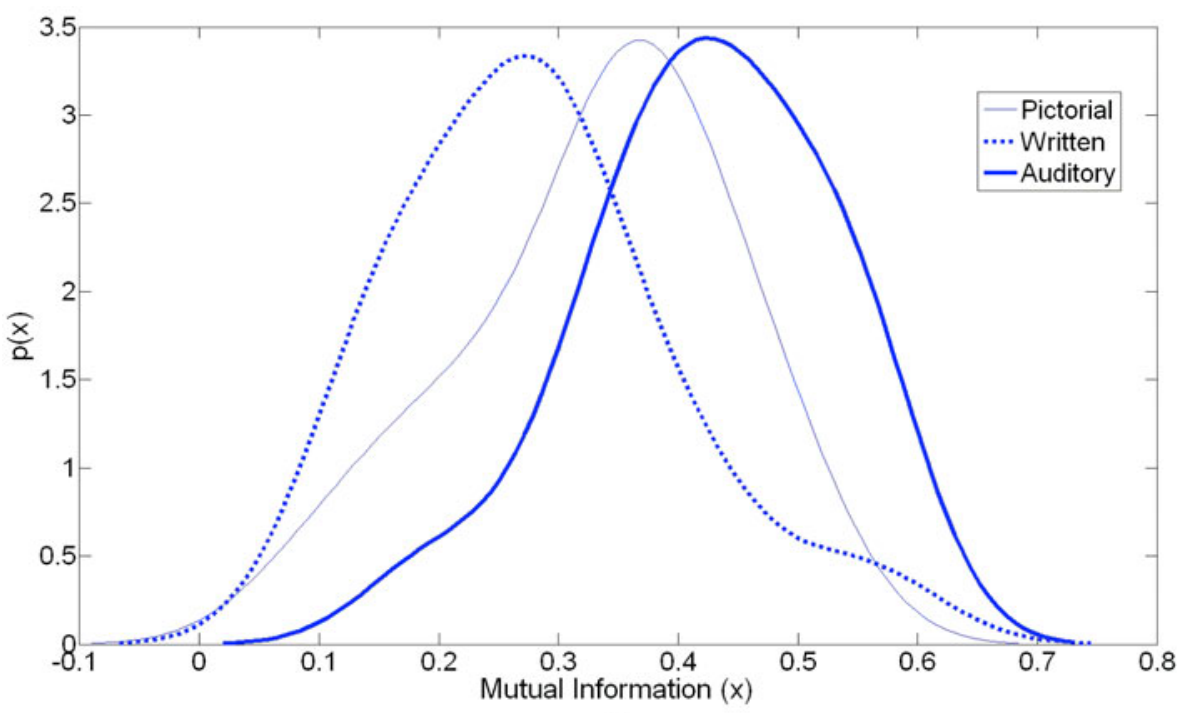

(a)

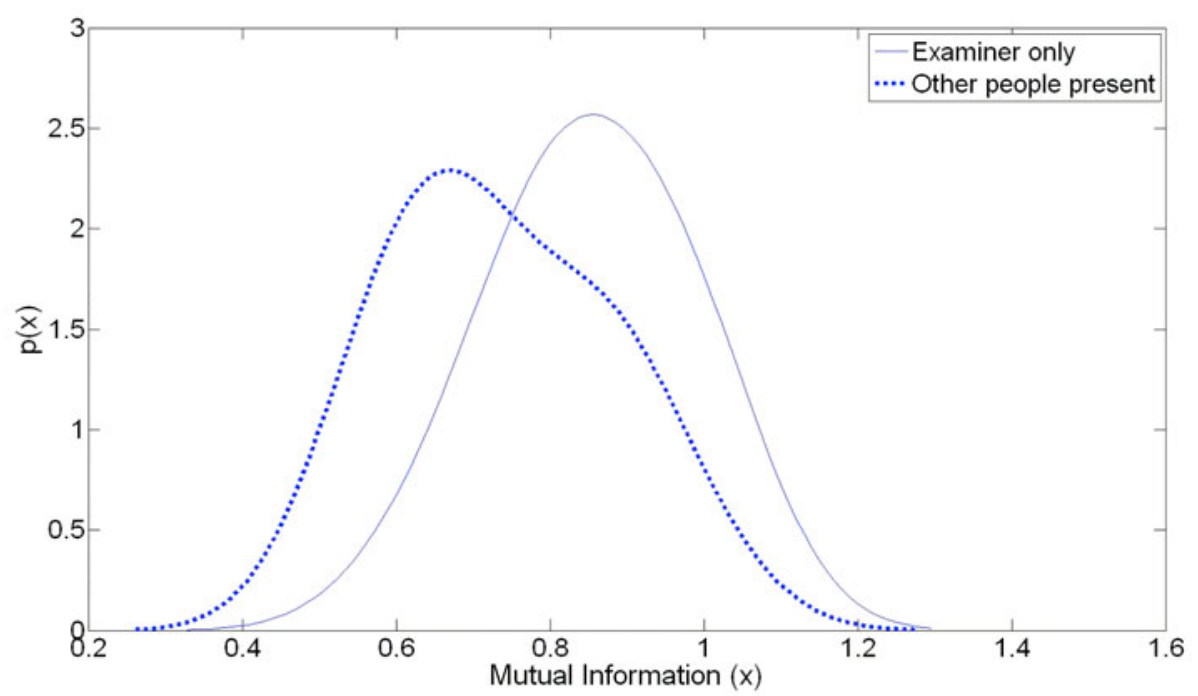

(b)

Fig. (4). Estimated probability densities of the mutual information for (a) presentation modality and (b) presence of people in the environment.

plying that for this particular subject, presentation modality is a neutral factor; neither the pictorial nor the written modality had an advantage over the other. From the above examples, it is evident that with mutual information, we can ascertain the role of each contextual factor (facilitating, hindering or neutral) for each subject, according to ICF prescriptions.

While the mutual information score is a single number, it allows us to gauge the explicit role of a contextual factor, even for those factors that have non-uniform effects across conventional performance measures. An example is the color factor, which improved sensitivity but lowered specificity for the majority of participants. Comparing the participants' mutual information values under no color and color conditions indicates that $67 \%$ of participants (participants 1, 2, 3, $6,9,10,11,12$ ) had better information transmission (higher mutual information) when the shapes were displayed to them without color. Hence, the color factor had an overall hindering effect on this population of participants.
Table 2. Effect of Stimulus Duration on Mutual Information of the Participant with Disability

\begin{tabular}{|c|c|c|}
\hline Experiments & $\mathbf{M I}_{\mathbf{6 0 0}}[\mathbf{b i t s}]$ & $\mathbf{M I}_{\mathbf{1 0 0 0}}[\mathbf{b i t s}]$ \\
\hline \hline $\begin{array}{c}\text { Presentation modality } \\
\text { Pictorial } \\
\text { Written } \\
\text { Auditory }\end{array}$ & $\begin{array}{l}0.1991 \pm 0.0521 \\
0.1974 \pm 0.0591 \\
0.4271 \pm 0.0950\end{array}$ & $\begin{array}{l}0.4690 \\
0.4690 \\
0.5436\end{array}$ \\
\hline $\begin{array}{c}\text { No prior knowledge (random } \\
\text { stimulus presentation) }\end{array}$ & $0.2289 \pm 0.0865$ & 0.8366 \\
\hline $\begin{array}{c}\text { No background noise (quiet } \\
\text { environment) }\end{array}$ & $0.1991 \pm 0.0521$ & 0.4690 \\
\hline Uncolored cues & $0.1687 \pm 0.0374$ & 0.5197 \\
\hline $\begin{array}{c}\text { No people in the environment } \\
\text { (other than examiner) }\end{array}$ & $0.7560 \pm 0.1256$ & 0.9982 \\
\hline
\end{tabular}

$\mathrm{MI}_{600}$ (Mean and Standard Deviation) and $\mathrm{MI}_{1000}$ Denote the Mutual information Corresponding to Stimulus Presentation Periods of 600 and 1000 Milliseconds. In all Cases, $\mathrm{MI}_{1000}>\mathrm{MI}_{600}, \mathrm{p}<0.05$ by a One-Sided, One-Sample t-Test. 


\subsection{Ranking Contextual Factors Based on the Signifi- cance of their Effect}

Standard statistical tests such as the analysis of variance (ANOVA) are not conducive to the ranking of independent variables (contextual factors) based on the significance of their effect on the dependent variables (performance measures). For example, both color and the presence of people in the environment had significant effects on switch activation specificity. It is unclear which effect was more influential. The mutual information measure however allows us to rank different contextual factors according to the strength of their effects. Returning to the example of individual effects of color and the presence of people in the environment we calculated the difference between a subject's mutual information with and without the presence of color and subsequently, the difference between a subject's mutual information in the absence and presence of people in the environment. Fig. (6) presents these mutual information deltas for all twelve participants. The figure suggests that the performance of $75 \%$ of the participants (participants 2, 4, 5, 6, 7, 8, $9,10,11)$ was affected more significantly by color than by the presence of people.

\subsection{Inter-Subject Comparison}

Mutual information addresses the problem of intersubject comparison. Assignment of a unifying quantitative measure to each participant provides the convenience of ranking participants based on the magnitude of impact they experienced from each factor.

\subsection{Objective Method of Performance Assessment for Users with Disability}

Results in Table 2 represent an example of how an individual's mutual information can be increased by modifying the characteristics of stimulus source (in this case, increasing the presentation period of each stimulus). This observation suggests another benefit of using the mutual information measure. When changing the physical characteristics of the communication channel is not possible (i.e. disability cannot be removed), an optimal information transmission rate can still be found by adjusting source characteristics (e.g. AAC display colors), receiver characteristics (e.g. changing the type of switch), or accounting for the effect of environmental and personal context (e.g. blocking out environmental noise or altering the presentation modality).

\subsection{Limitations of Present Study}

The goal of this study was to demonstrate the use of mutual information as a measure of contextual effects on singleswitch use, rather than to definitively establish specific contextual effects. In terms of the latter topic, several improvements can be made in subsequent studies. For example, one could go beyond the univariate analyses presented here and explore possible interaction effects between multiple contextual factors through additional data collection and multivariate analyses. The number of participants can be increased to provide a stronger basis for identifying the main role of each contextual factor. Mutual information reliability has only been considered in one case and should be further investigated in future studies. In particular, the effect of time, a central contextual factor, can also be analyzed.

\section{CONCLUSION}

In this paper, we proposed the use of mutual information as a quantitative method for measuring the impact of context, as defined by the World Health Organization's ICF model, on information transmission within a single-switch paradigm. Using empirical data from a single mechanical switch experiment, we demonstrated that mutual information can provide: (a) an objective way to determine the ICF classifi-

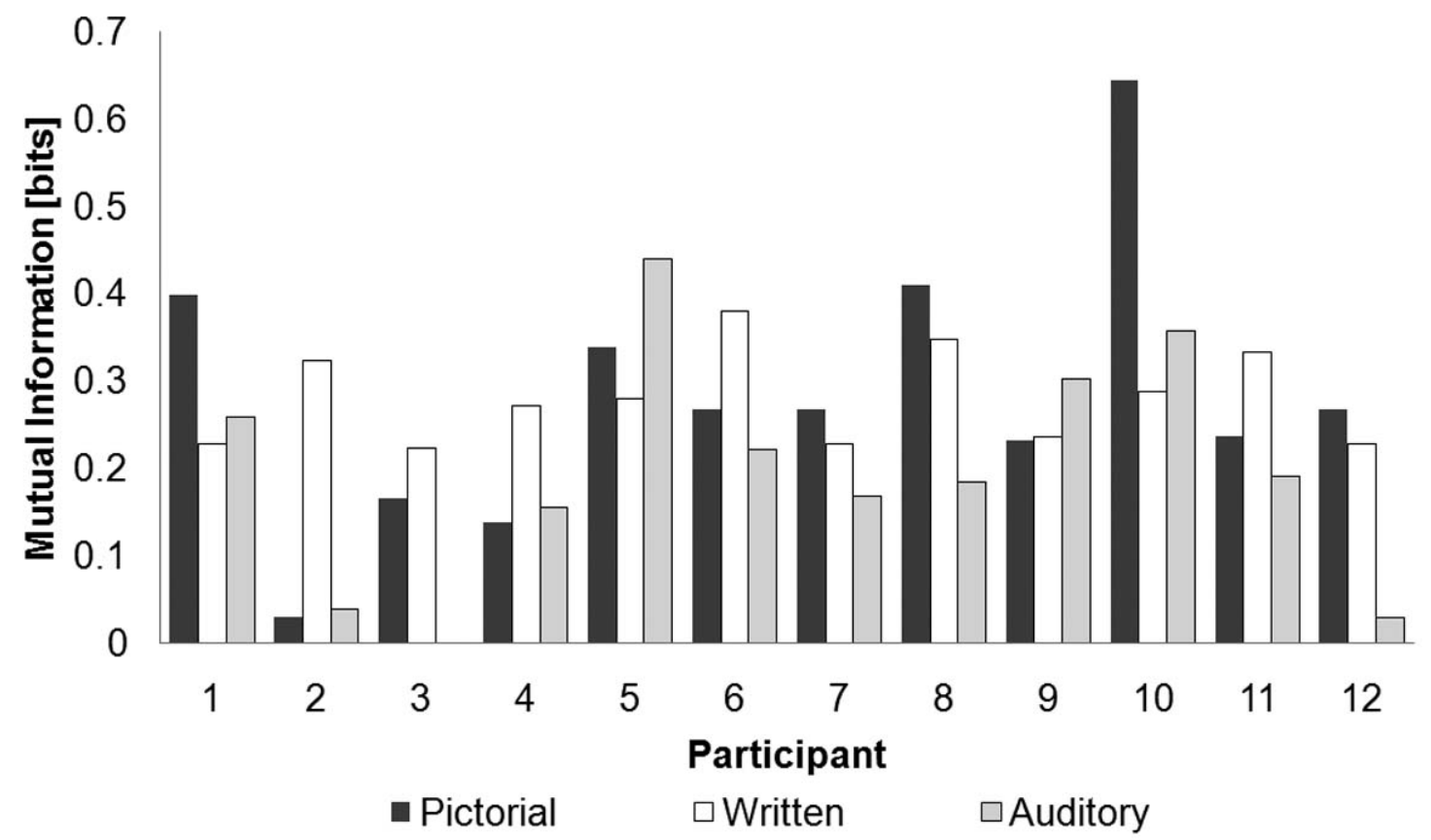

Fig. (5). Participants' mutual information in response to pictorial (dark bars), written (unshaded bars) and auditory stimuli (grey bars). 


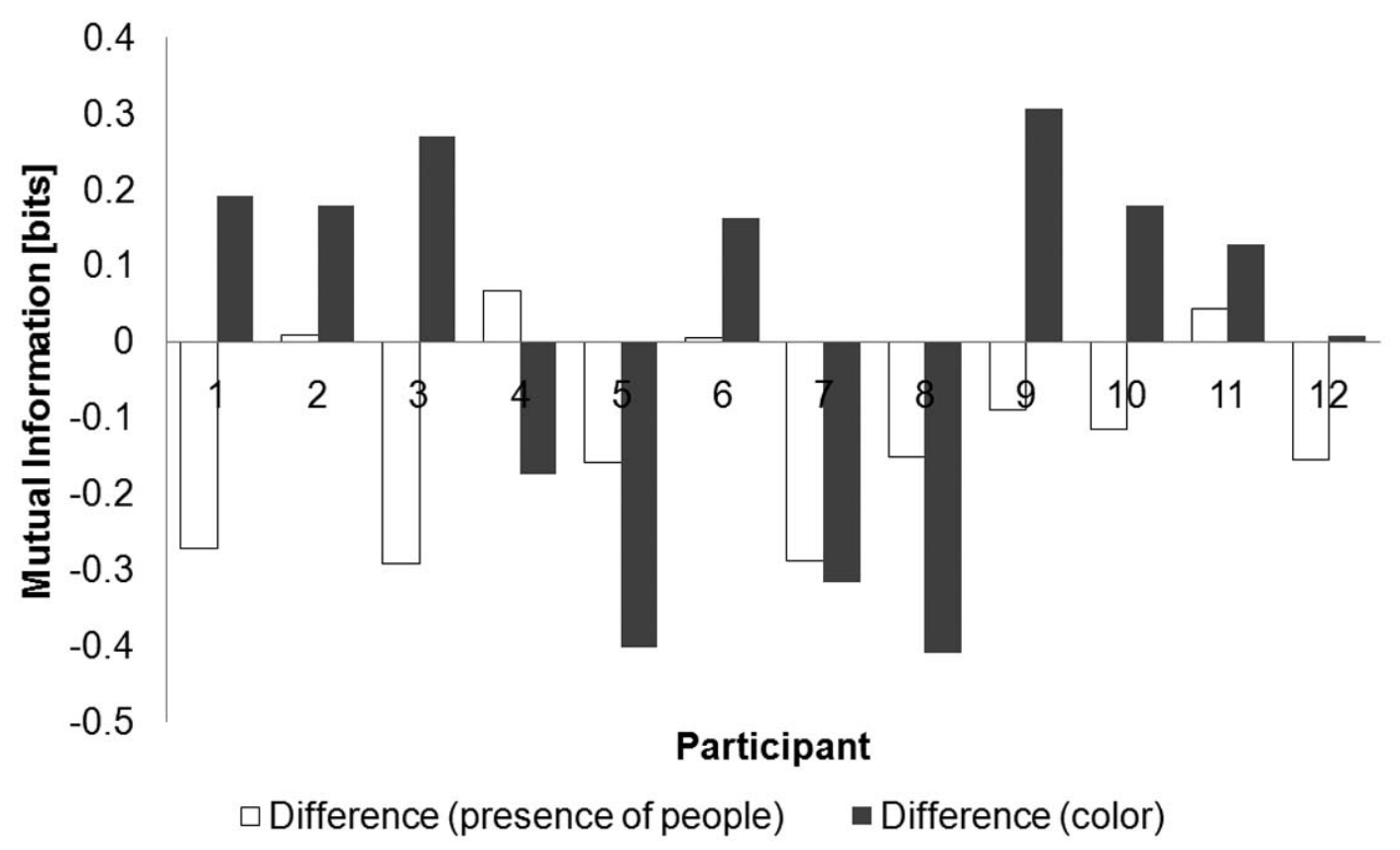

Fig. (6). Difference in participants' mutual information with and without the following contextual factors: presence of people (unshaded bars) and color (shaded bars).

cation of contextual factors (i.e., facilitator, barrier or neutral), (b) the ability to rank different contextual factors according to the strength of their individual effects on the performance of a given user, (c) a means of consolidating nonuniform effects of a particular contextual factor into a unique measure, and (d) the ability to rank different individuals based on their mutual information for a given task. This measure may be particularly useful as an objective means of establishing optimal information transmission rates in individuals with disability.

\section{ACKNOWLEDGEMENTS}

This research has been supported by a grant and a postgraduate scholarship from the Natural Sciences and Engineering Research Council of Canada and a Whipper Watson scholarship from Bloorview Kids Rehab, Toronto, Canada.

\section{REFERENCES}

[1] Tai K, Blain S, Chau T. A review of emerging access technologies for individuals with severe motor impairments. Assist Technol 2008; 20: 204-19.

[2] Blain S, Chau T, Mihailidis A. Peripheral autonomic signals as access pathways for individuals with severe disabilities: a literature appraisal. The Open Rehab J 2008; 1: 27-37.

[3] Cook AM, Hussey SM. Assistive technologies: principles and practice. $2^{\text {nd }}$ ed, St. Louis, MO, USA: Mosby Inc. 2002.

[4] World Health Organization. International Classification of Functioning, Disability \& Health: ICF. Geneva, Switzerland: World Health Organization 2001.

[5] Cykes C. Health Classifications 1 -An introduction to the ICF. World Confederation for Physical Therapy Keynotes 2006 [Accessed: 2007 September]. Available from: http://www.wcpt.org/ common/docs/keynotes/ICFIntro.pdf

[6] Bornman J. The World Health Organization's terminology and classification: application to severe disability. Disabil Rehabil 2004; 26(3): 182-8.

[7] Blackhurst AE. A functional approach to the delivery of assistive technology services. National Assistive Technology Research Insti- tute 2001 [Accessed 2007 September]. Available from: http://natri. uky.edu/resources/fundamentals/function.html

[8] Blackhurst AE, Lahm EA. Technology and exceptionality foundations. In: Lindsey JD, Ed. Technology and exceptional individuals. $3^{\text {rd }}$ ed, Austin, TX: ProEd 2000; pp. 3-45.

[9] Haley SM, Coaster WJ, Binda-Sundberg K. Measuring physical disablement: the contextual challenge. Phys Ther 1994; 74(5): 443 51 .

[10] Mihaylov SI, Jarvis SN, Colver AF, Beresford B. Identification and description of environmental factors that influence participation of children with cerebral palsy. Dev Med Child Neurol 2004; 46: 299304.

[11] Lawlor K, Mihaylov S, Welsh B, Jarvis SJ, Colver AF. A qualitative study of the physical, social and attitudinal environments influencing the participation of children with cerebral palsy in northeast England. Pediatr Rehabil 2006; 9(3): 219-28.

[12] Colver A. Study protocol: SPARCLE- a multi-center European study of the relationship of environment to participation and quality of life in children in cerebral palsy. BMC Public Health 2006; 6(105): 10

[13] Craig A, Tran Y, McIsaac P, Boord P. The efficacy and benefits of environmental control systems for the severely disabled. Med Sci Monit 2005; 11(1): 32-9.

[14] Wang PP, Badley EM, Giganac M. Exploring the role of contextual factors in disability models. Disabil Rehabil 2006; 28(2): 135-40.

[15] Edwards E. Information transmission, an introductory guide to the application of the theory of information to the human sciences. London, UK: Chapman and Hall 1969.

[16] Shannon CE. A mathematical theory of communication. Bell System Tech J 1948; 27: 379-423, 623-56.

[17] Hick WE. On the rate of gain of information. Q J Exp Psychol 1952; 4: 11-26.

[18] Hyman R. Stimulus information as a determinant of reaction time. J Exp Psychol 1953; 45: 188-196.

[19] Klemmer ET, Frick FC. Assimilation of information from dot and matrix patterns. J Exp Psychol 1953; 45: 15-9.

[20] Fitts PM. The information capacity of the human motor system in controlling the amplitude of movement. J Exp Psychol 1954; 47: 381-91.

[21] Broadbent DE. Perception and Communication. London, UK: Pergamon Press 1958. 
[22] Ellison KW, Buckout R. Psychology and criminal justice. New York, NY, USA: Harper \& Row 1981.

[23] Wells GL. What do we know about eyewitness identification? Am Psychol 1993; 48: 553-71.

[24] Wright DB, Davies GM. Eyewitness testimony. In: Durso F, Ed. Handbook of Applied Cognition, West Sussex, UK: John Wiley \& Sons 1999.

[25] Attneave F. Applications of information theory to psychology (A summary of basic concepts, methods and results). New York, NY, USA: Henry Holt and Company Inc. 1959.

[26] Wickens CD, Hollands JG. Engineering Psychology and Human Performance. $3^{\text {rd }}$ ed. Upper Saddle River, NJ, USA: Prentice-Hall Inc. 2000.

[27] Card SK, Moran TP, Newell A. The Psychology of HumanComputer Interaction. Hillsdale, NJ, USA: Lawrence Erlbaum Associates Inc. 1983.

[28] Landauer TK, Nachbar DW. Selection from alphabetic and numeric menu trees using a touch screen: Breadth, depth, and width, Proceedings of the CHI '85 Human Factors in Computing Systems, ACM, New York, NY 1985; pp. 73-8.

[29] Olson JR, Nilsen E. Analysis of the cognition involved in spreadsheet software interaction. Human-Computer Interaction 1988; 3 : pp. 309-50.

[30] Seow SC. Information theoretic models of HCI: A comparison of the Hick-Hyman law and Fitt's law. Human-Computer Interaction 2005; 20: pp. 315-52.

[31] Chan RB, Childress DS. On a unifying noise-velocity relationship and information transmission in human machine systems. IEEE Trans Syst Man Cybern 1990a; 20(5): 1125-35.
[32] Chan RB, Childress DS. On information transmission in humanmachine systems: channel capacity and optimal filtering. IEEE Trans Syst Man Cybern 1990b; 20(5): 1136-45.

[33] Ogawa K. An evaluation method of computer usability based on human-to-computer information transmission model. Ergonomics 1992; 35(5 \& 6): 577-90.

[34] Poock GK, Blackstone SW. Using information theory to measure effectiveness of an augmentative and alternative communication display. Augment Altern Commun 1992; 8(4): 287-96.

[35] Sanger TD, Henderson J. Optimizing assisted communication devices for children with motor impairments using a model of information rate and channel capacity. IEEE Trans Neural Syst Rehabil Eng 2007; 15(3): 458-68.

[36] Cover TM, Thomas JA. Elements of Information Theory. $2^{\text {nd }}$ ed, Hoboken, NJ, USA: John Wiley and Sons Inc. 2006.

[37] Pierce JR. An introduction to information theory: symbols, signals $\&$ noise. $2^{\text {nd }}$ ed, New York, NY, USA: Dover, Publications 1980.

[38] Roman S. Coding and information theory. New York, NY, USA: Springer, 1992.

[39] Lathi BP. Modern digital and analog communication systems. 3rd ed, New York, NY, USA: Oxford University Press 1998

[40] Haykin S. Communication Systems. $4^{\text {th }}$ ed, New York, NY, USA: John Wiley and Sons 2001.

[41] Van Trees HL. Detection, estimation, and modulation theory, Part I. New York, NY, USA: John Wiley and Sons Inc. 2001.

[42] Laming D. Information theory of choice-reaction times. New York, NY, USA: Academic Press 1968.

(C) Memarian et al.; Licensee Bentham Open.

This is an open access article licensed under the terms of the Creative Commons Attribution Non-Commercial License (http: //creativecommons.org/licenses/by$\mathrm{nc} / 3.0 /$ ) which permits unrestricted, non-commercial use, distribution and reproduction in any medium, provided the work is properly cited. 